- organization and scientific scope to the US National Institute of Mental Health, a major US brain-science funder, although on a smaller scale.

The Chinese centre will be a partnership between Beijing's premier biomedical institutions, among them the Chinese Academy of Sciences, the Academy of Military Medical Sciences, Peking University and Tsinghua University. Luo says it will support projects that use the latest biomedical methods, such as highthroughput single-gene sequencing, precision genome editing and big-data processing. $\mathrm{He}$ also hopes to develop better imaging tools, including a voltage sensor that can directly record neuronal activity, and high-speedimaging microscopes that will allow detailed views of brain activity.

This year, Luo plans to use 180 million Chinese yuan (US\$29 million) provided by the Beijing municipal government to hire the first five or six research groups, and to install them in a building already constructed by the municipality, which is across the road from his institute. When operating at its full capacity of 50 researchers, which Luo plans to have within 5 years, some 400 million yuan per year will be needed. He hopes to secure this from the brain-science project, with a substantial amount still coming from Beijing.

Luo says that it will be a "docking site" for the Chinese brain project, which has been in planning since the United States and Europe launched their programmes. So far, few firm details about the project have been released. Scientists who spoke to Nature say they expect that the government will officially launch the initiative some time this year.

\section{STAFFING CHALLENGES}

In the meantime, other facilities are preparing their bids for support from the national project. A large science park under construction in Shanghai will house a 'southern centre' for neuroscience research. The centre's organizers say this will support many more principal investigators than its Beijing counterpart, which scientists are dubbing the northern centre.

Feng Jianfeng, a computational biologist and head of Fudan University's Institute of Science and Technology for Brain-inspired Intelligence, has been involved in organizing the Shanghai projects. He says that one focus will use artificial intelligence (AI) to study brain diseases. Feng adds that, with 190 million yuan from the university, he is already setting up a brain-imaging facility that will house the largest number of magnetic resonance imaging devices in Asia, and will be based at the southern centre. AI algorithms will screen the images, comparing diseased brains with healthy ones, to form part of the world's largest brain database, he says.

Another programme expected to be integral to the country's brain-science initiative is an international connectome project, which is being designed by $\mathrm{Mu}$-Ming Poo, director of the Institute of Neuroscience in Shanghai. Connectome projects attempt to map out all the neural connections in the brain.

Finding enough researchers might be the greatest challenge for both the individual centres and the Chinese brain-science project. Jeffrey Erlich, a neuroscientist at NYU Shanghai, says that, as well as hiring top neuroscientists, the initiatives will need to fund postdoctoral positions and graduateschool research posts offering internationally competitive salaries.

"That would increase the number of top students going into neuroscience," says Erlich. "Then, in five to ten years, China could have a fresh crop of top young scientists." -

\title{
Exoplanet hunter will seek worlds close to home
}

\section{NASA's mission is designed to spot planets orbiting nearby bright stars.}

\section{BY ALEXANDRA WITZE}

$\mathrm{F}$ illing the shoes of NASA's Kepler spacecraft won't be easy. Since its launch in 2009, Kepler has discovered nearly threequarters of the 3,700-plus known exoplanets. And there are thousands more candidates waiting to be confirmed.

So NASA is taking a different approach with its next planet-hunting mission. On 16 April, the agency plans to launch the US\$337-million Transiting Exoplanet Survey Satellite (TESS), which will scrutinize 200,000 nearby bright stars for signs of orbiting planets. TESS will probably find fewer worlds than Kepler did, but they are likely to be more important ones.

"It's not so much the numbers of planets that we care about, but the fact that they are orbiting nearby stars," says Sara Seager, an astrophysicist at the Massachusetts Institute of Technology (MIT) in Cambridge and deputy science director for TESS.
TESS is meant to identify planets that are close enough to Earth for astronomers to explore them in detail. Team scientists estimate that the spacecraft will discover more than 500 planets that are no more than twice the size of Earth (P. W. Sullivan et al.

\section{"We'll see a whole new opening of exoplanet studies."} Astrophys. J. 809, 77; 2015). These worlds will form the basis for decades of further studies, including searches for signs of life. "We'll see a whole new opening of exoplanet studies," Seager says.

Both Kepler and TESS are designed to scan the sky for planetary transits, the slight dimming that occurs when a planet moves across the face of a star and temporarily blocks some of its glow. For most of its mission, Kepler stared at a deep but narrow slice of the Universe - peering out some 920 parsecs (3,000 light years) from Earth but covering only $0.25 \%$ of the sky. Its celestial census showed that planets were common throughout the Milky Way. "We found that planets are everywhere," says Elisa Quintana, an astrophysicist at NASA's Goddard Space Flight Center in Greenbelt, Maryland.

\section{MEETING THE NEIGHBOURS}

By contrast, TESS will go shallow and broad - looking at stars within 90 parsecs of Earth but covering more than $85 \%$ of the sky. Its 4 cameras will give the spacecraft a field of view about 20 times the size of Kepler's (see 'Scanning the sky'). TESS will sweep the southern sky first and then, after a year, turn its attention to northern stars.

The observing swathes in each hemisphere will overlap at the south and north ecliptic poles, which are points perpendicular to the plane of Earth's orbit. That's by design, because NASA's James Webb Space Telescope, now planned for a 2020 launch, will also be able to study those regions at any 


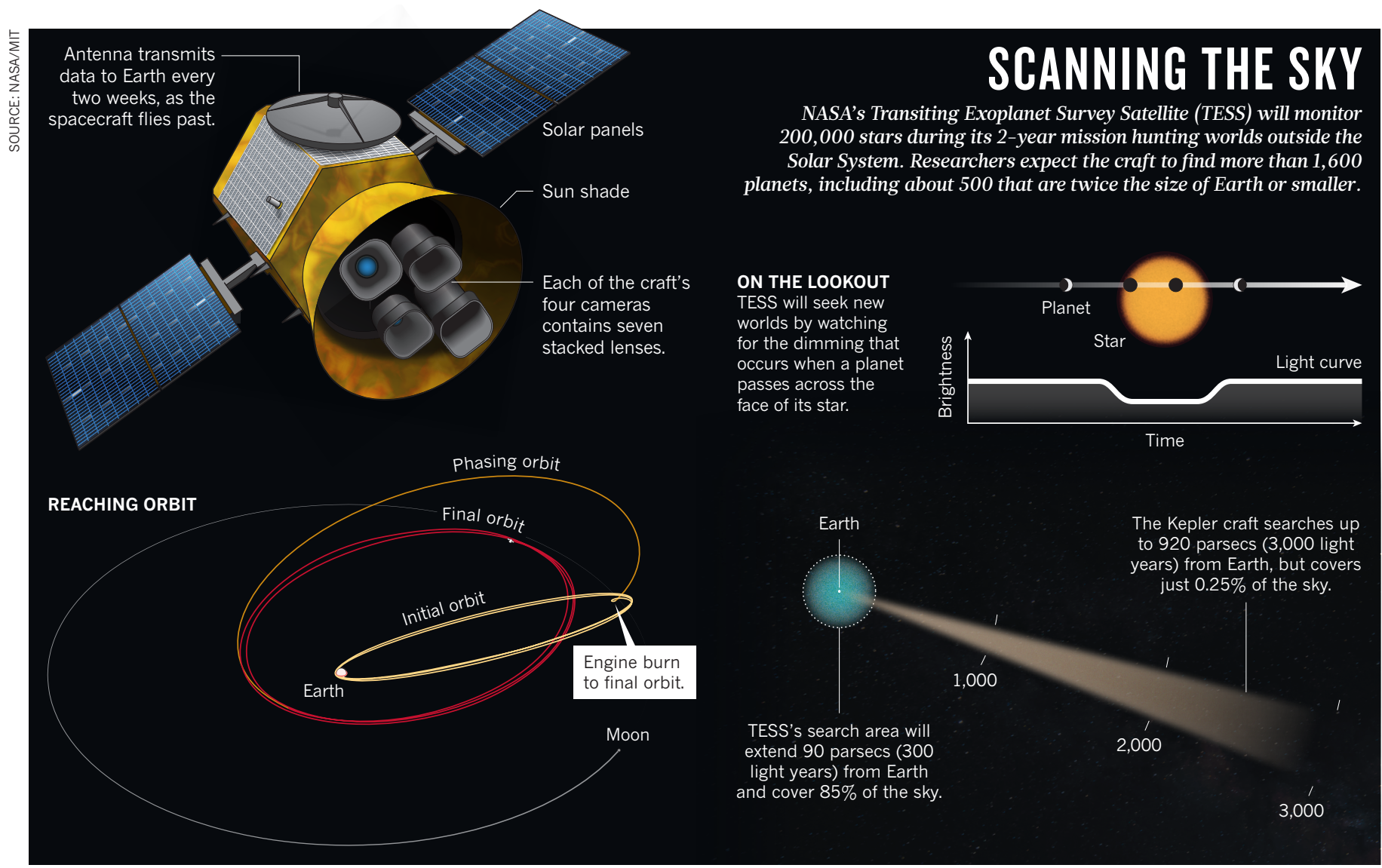

given time. Webb's 6.5-metre primary mirror will allow detailed spectroscopic studies of the planets' atmospheres, but it will be in high demand for a range of other astronomical research. "The time on Webb is going to be so precious," says George Ricker, an astrophysicist at MIT and TESS's principal investigator.

Once TESS spots interesting planetary candidates, a fleet of Earth-based observatories will kick into action to gather more data. These

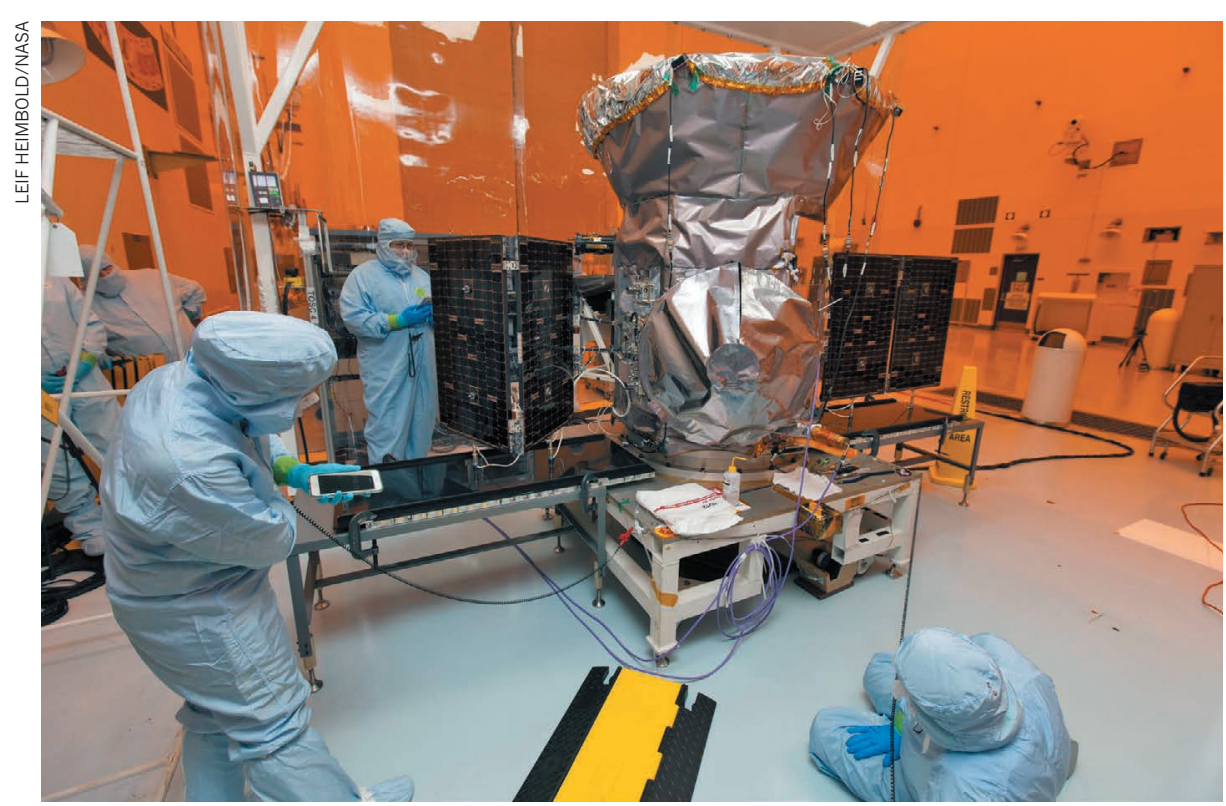

The Transiting Exoplanet Survey Satellite will search more than $85 \%$ of the sky.

will include planet-hunting stalwarts such as the HARPS instrument at the European Southern Observatory in La Silla, Chile, and the new Miniature Exoplanet Radial Velocity Array (MINERVA)-Australis, a group of five planned 0.7-metre telescopes near Toowoomba, Australia. "We have the ability to hammer on a target every night if we need to," says Rob Wittenmyer, an astronomer at the University of Southern Queensland in Toowoomba who helps lead MINERVA-Australis.
These and other ground-based telescopes will be able to deduce the TESS planets' masses, and from that their composition - whether they are rocky, icy, gassy or something else.

\section{A WHOLE NEW WORLD}

Recent research suggests that TESS may yield a greater bounty than once thought. Earlier this year, MIT astronomer Sarah Ballard recalculated how many planets TESS might find orbiting the cool, plentiful stars known as M dwarfs - and predicted some 990 such planets, 1.5 times more than earlier estimates (S. Ballard Preprint at https:// arxiv.org/abs/1801.04949; 2018). The sheer volume of discoveries would allow astronomers to begin comparing broad classes of exoplanets: learning how stellar flares affect planetary atmospheres, for instance, or what sorts of planets surround stars of different ages.

TESS will soon have company. The European Space Agency (ESA) plans to launch its Characterising Exoplanet Satellite late this year. The craft will measure the sizes of known planets - from those a little bigger than Earth to ones that are roughly Neptune-sized orbiting nearby bright stars. ESA is also planning two missions for the 2020s: PLATO to study Earth-sized exoplanets, and ARIEL to study planetary atmospheres.

The next generation of missions will come just in time: Kepler is on its last legs, with only a few months' worth of fuel left to help it make its final discoveries. 